\title{
Optical and mechanical design of a straylight rejection baffle for COROT
}

\author{
J.Y. Plesseria ${ }^{*}$, E. Mazy ${ }^{\mathrm{a}}$, J.M. Defise ${ }^{\mathrm{a}}$, P. Rochus ${ }^{\mathrm{a}}$, E. Lemmens ${ }^{\mathrm{b}}$, D. Vrancken ${ }^{\mathrm{b}}$ \\ ${ }^{a}$ Centre Spatial de Liège, Université de Liège, av. du Pré-Aily, B-4031, Angleur Belgium \\ ${ }^{\mathrm{b}}$ Verhaert Design and Development, Hogenakkerhoekstraat, 9, B9150, Kruibeke, Belgium
}

\begin{abstract}
The COROT mission is part of the program "Petites Missions" of CNES (French space agency). It implies international cooperation between France, Belgium, Germany, Austria, Spain and the European Space Agency (ESA). COROT aims to perform astroseismology measurements and to detect exoplanets. Long duration observations of stars will be used to detect periodic variations with an afocal telescope followed by a dioptric objective and 4 CCDs.

Due to the orbit of the spacecraft (low altitude polar orbit) and even if the observation are performed in a direction perpendicular to orbit plane, the measurements can be disturbed by the straylight reflected on the earth (albedo) that can generate periodic perturbation.

CSL is in charge of the design and procurement, with the help of Belgian industries, of a baffle and its protective cover that will be mounted on top of the afocal entrance telescope. The requirements are very stringent from the optical point of view as well as from the mechanical point of view.

The rejection of the baffle must be of the order of $10^{13}$ for field angles above 20 degrees while the allocated mass is 19 kilograms.
\end{abstract}

Keywords: Astroseismology, exo-planets, straylight, COROT, baffle

\section{INTRODUCTION}

The COROT mission is based on the observation during periods up to five months of a group of stars. The variations of measured intensity will inform scientists of the seismic oscillations of stars or can indicate the transit of a planet. Separation between both effects is made by the use of a prism that can determine the spectral distribution of the variation.

The location of the spacecraft in low earth orbit is the driving parameter for the design of the baffling system. The light reflected by Earth albedo will vary during each revolution, this periodic perturbation will disturb the measurement of the periodic variation of star intensity.

A first design of the telescope, including the baffle, has been performed under CNES responsibility taking into account the envelope constraint of the launcher and optical requirements. This study led to an afocal telescope followed by a dioptric objective and a so called 1.5-stage baffle.

At the beginning of the international collaboration, the design of the baffle was transmitted to CSL. Further evolutions have been implemented to improve the interface with the telescope.

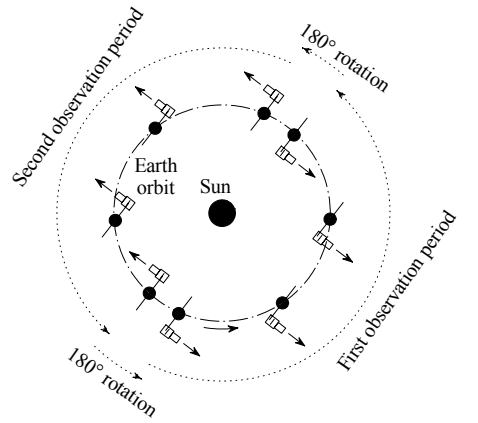

\section{MISSION OVERVIEW}

The orbit selected for COROT is an inertial polar orbit. By observation perpendicular to the plane of the orbit, it will allow permanent observation of a determined field with no occultation from Earth. During a complete observation ( $\sim 150$ days), the Sun will stay behind the aperture. At the end of the observation period, before the Sun goes in the forward plan, the spacecraft will rotate by $180^{\circ}$ to observe in the other direction. As a result, the Sun will again stay behind.

Around rotation of the spacecraft, some shorter observations will be performed.

$\leftarrow$ Figure 1: COROT observation principle

*iyplesseria@ulg.ac.be; phone +32.4.367.66.68; fax +32.4.367.56.13; www.ulg.ac.be/cslulg 


\section{SPACECRAFT OVERVIEW}

\subsection{Service module (PROTEUS)}

The platform used for this spacecraft is the generic service module PROTEUS proposed by Alcatel Space (France). This module is designed to implement small payload $(300 \mathrm{~kg})$. It includes power supply, S-band telemetry and attitude control. Solar panels are mounted on both sides of the platform.

In this particular mission, startrackers are required to increase the stability and pointing accuracy of the telescope. These startrackers are mounted on the payload but are used by the attitude control system of the platform.

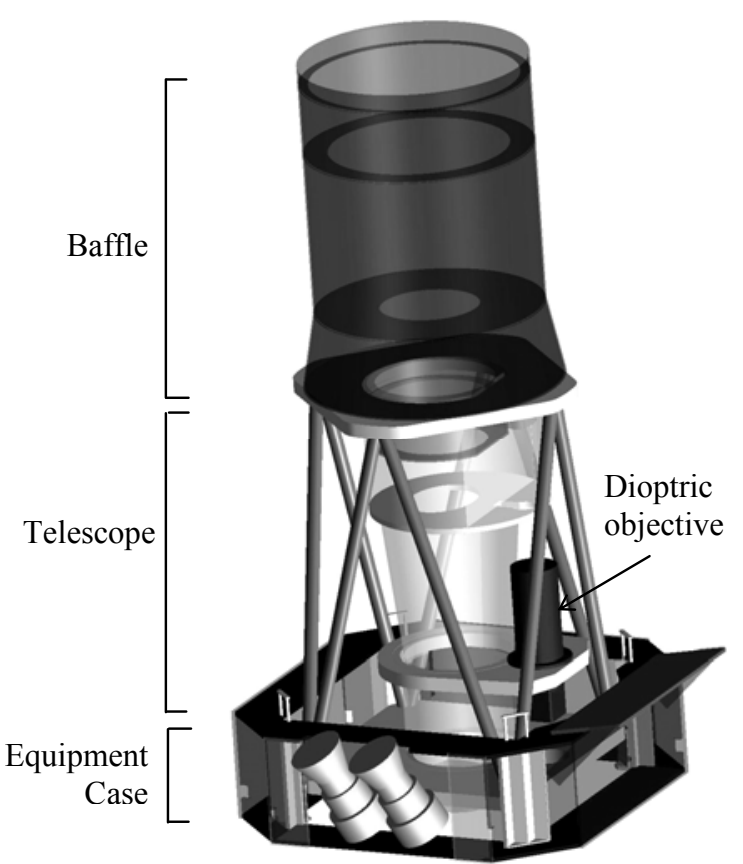

\subsection{Equipment case (COROTCASE)}

The equipment case is mounted on top of the platform. The structure of this element is designed and manufactured in Belgium. It contains all electronic boxes required for the acquisition and pre-treatment of the images taken by the detectors as well as for the thermal control. This case also maintains the startrackers.

\subsection{Camera (COROTCAM)}

The camera is the focal plane assembly containing the 4 detectors ( 2 for astroseismology and 2 for exoplanets detection) and the read-out electronic. The focal plane assembly also contains a prism in the exoplanets channel.

The camera includes the dioptric objective focusing the collimated beam coming out of the afocal telescope onto the detectors.

\subsection{Telescope (COROTEL)}

COROTEL is under the responsibility of the Astrophysics Laboratory of Marseille (LAM). It includes the telescope itself but also the baffle and cover. The telescope and the optics are manufactured in France while the baffle and cover will be provided by Belgium under the responsibility of CSL.

Figure 2: COROT payload (without cover)

\section{BAFFLE DESCRIPTION}
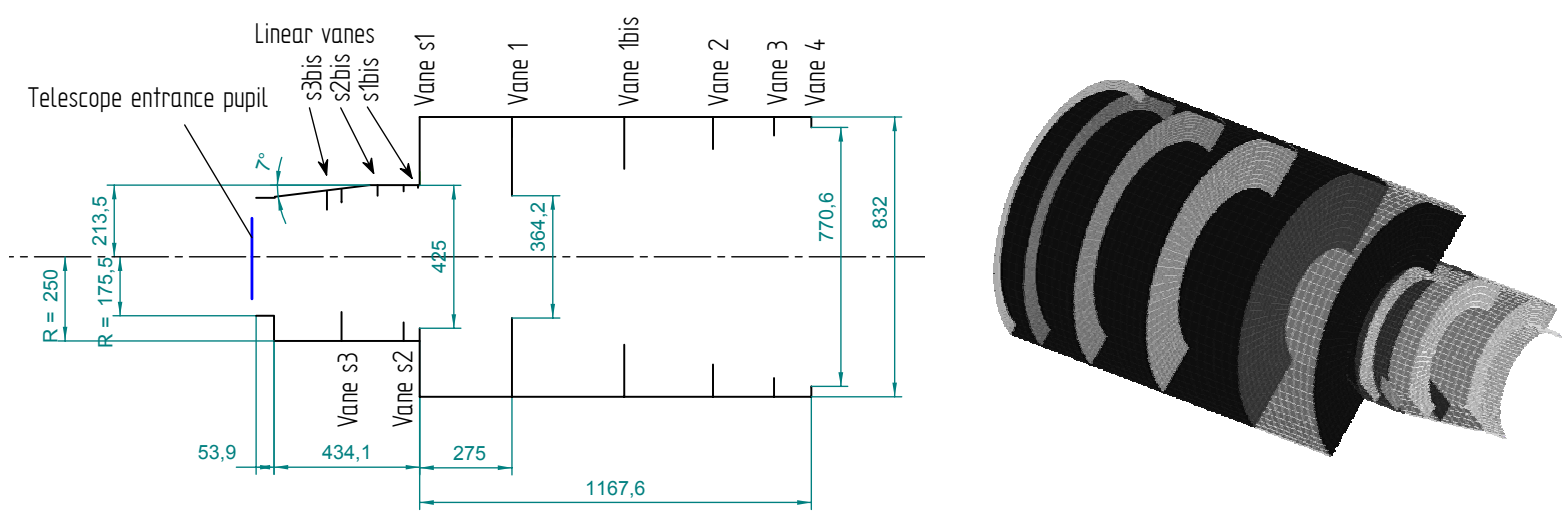

Figure 3: Optical design of the COROT baffle 
The aim of the baffle is to attenuate the straylight coming from sources above 20 degrees. This value has been chosen to allow some off pointing with respect to perpendicular to orbit and thus to increase the number of potential observation targets.

A very first baffle design, optimised using ASAP and APART, has been proposed by CNES/LAM at the beginning of the collaboration. Some mechanical constraints have appeared and some modifications were required, mainly in the second (smaller) tube due to the chamfer needed for the accommodation of the secondary mirror. The modifications have been proposed by CNES/LAM (responsible for the overall instrument straylight performances) and this new design has been analysed by CSL using ASAP.

A baffle made of two stages would have been ideal. The first stage would have prevented any light above 20 degrees to reach the second stage. Unfortunately, the envelope problem linked to the size of the possible fairing imposes a reduction to a 1.5 stage baffle (separation being at vane 1). It means that the light up to 32.5 degrees enters the second stage and the light between 20 and 32.5 degrees is absorbed in the second stage. It has the advantage on a one-stage baffle that the total straylight is attenuated in two different optimised elements.

The resulting baffle consists in two consecutive tubes containing a total of 11 vanes as shows on Figure 3.

\section{INITIAL STRAYLIGHT ANALYSIS}

\subsection{First order analysis (vanes)}

In the case of COROT, the most critical path is the one by which the light is reaching the primary mirror after only one scattering on surfaces. Once on the mirror, this light is scattered by contamination and roughness directly within the field of view and goes to the detector without further attenuation. A first order analysis has been run with Matlab in order to detect any surface that can be lightened by external source and that has a direct view factor to the primary mirror.

In the Matlab procedure written to perform this analysis, a matrix of points is drawn on the primary mirror. Then, for any observation point in space, the number of point of the mirror matrix seen without obstruction is counted. The ratio of the number of visible points to the total number of points gives the portion of primary mirror seen.

This computation can be performed for any point in the baffle. Different procedures have been written to automatically perform these computations in the planes of the vanes. By comparing the surfaces that view the mirror with the vanes, we can verify the critical surfaces.

Figure 4 and Figure 5 are examples of the result obtained. The grey levels indicate the percentage of primary mirror seen from black (mirror not seen) to white (full mirror seen). The two circles indicate the tube diameter and the edge of the vane. These two pictures indicate the situation in the plane of chicane 1 and chicane 4 . It clearly shows that the edge of chicane 1 is a critical element since it can be lightened by external light and directly see almost $80 \%$ of the primary mirror. Chicane 4 is not a problem since positive margin exists.

Rapid drawing analysis shows that the edge of chicane 1 is visible for luminous object up to 32.5 degrees from optical axis.
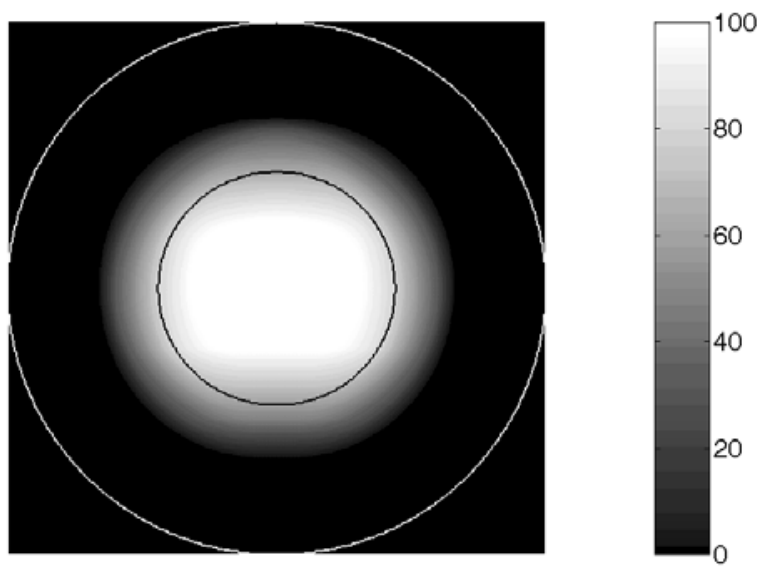

Figure 4: Chicane 1, the edge is a critical path because it sees about $80 \%$ of primary mirror

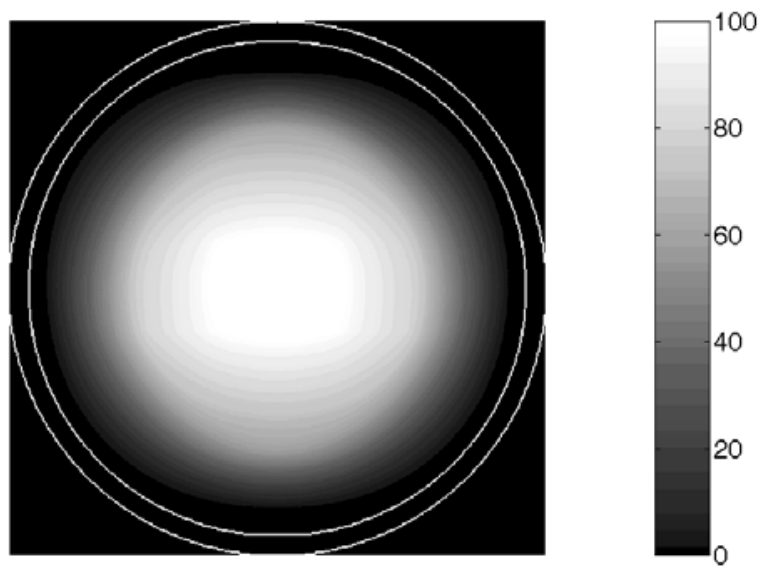

Figure 5: Chicane 4, the edge is not seen by primary mirror. 
Table 1 summarises the results of the computations. In addition to some of the edges that can be considered as critical at first order, other surfaces (back of vanes S3bis, S3, S2 and 1) are second order critical surfaces. The light can go deeper in the baffle, be scattered back to the vane rear face and than scattered again towards primary mirror. These paths added one attenuation factor (due to scattering) and will be taken into account in the global straylight analysis.

\begin{tabular}{|c|c|c|}
\hline Vane \# & Critical & $\begin{array}{c}\text { Field of the } \\
\text { sources }\end{array}$ \\
\hline S3bis & Yes $(50 \%)$ & $<20^{\circ}$ \\
\hline S3 & Yes $(80 \%)$ & $<21.5^{\circ}$ \\
\hline S2bis & No & - \\
\hline S2 & Yes (25\%) & $<25.5^{\circ}$ \\
\hline S1bis & No & - \\
\hline S1 & No & - \\
\hline 1 & Yes $(80 \%)$ & $<32.5^{\circ}$ \\
\hline 1bis & No & - \\
\hline 2 & No & - \\
\hline 3 & No & - \\
\hline 4 & No & - \\
\hline
\end{tabular}

Table 1: First order analysis of vanes

\subsection{First order analysis (tubes)}

The tubes are never seen by the primary mirror, nevertheless, we decided to further analyze the second (smaller) tube which parts can be second order critical surfaces. If they are too small, it is possible that the ray-tracing computation will not be able to detect them. We consider in this case that the light entering the telescope entrance pupil will be scattered once on the internal tube then will go on the primary mirror. For that, the Matlab procedure is modified and instead of placing a matrix on the primary mirror, it is placed once on the entrance pupil and once on the baffle aperture. Moving the observation point on the small tube surface for the two cases will determine which are the surfaces seen by the aperture and by the pupil.

Figure 6 shows the product of the surface viewed by the pupil by the surface viewed by the aperture of the baffle. This picture shows that the only non-null area is very close to the pupil and for very low field of view $\left(<20^{\circ}\right)$. This is considered not critical (unknown about the real arrangement of the connection between baffle and telescope tube could invalidate any conclusion in this area).
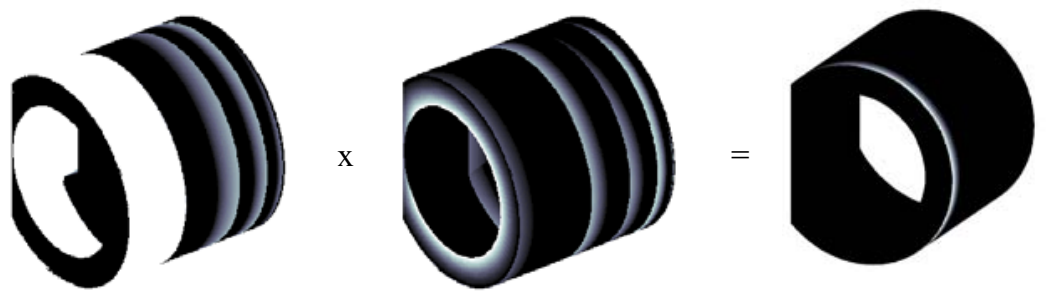

Figure 6: Detection of critical surfaces in the small tube (view from pupil times view from aperture). Only critical area is close to pupil (front of the picture)

As a conclusion, no critical surface exists in the second tube at first order. At the second order, the area is very marginal and for low field $\left(<20^{\circ}\right)$.

\subsection{ASAP modelling}

Following first (and partial second) order analysis, we can go to a ray-tracing model for higher level analysis with the following observation: the only critical surfaces with small area are the edges. It means that they must be analyzed separately (see 5.6).

ASAP model is a geometric one, it means that no thickness has been given for the walls except for the vanes (40 $\mu \mathrm{m})$. The junctions between all elements are supposed perfect. The modeling made by CSL should have been only done on the 
baffle itself but in this case, the results would have been hardly understandable in terms of performances for scientific observation. It has been decided to include in the model the afocal telescope (including entrance and exit pupil and intermediate focus) and the optical cavities of these two mirrors (they play an important role in the global straylight).

The output of the computation is thus the straylight coming out of the afocal telescope and entering the dioptric objective. This can also be split in straylight in the field of view that will go directly on the detector and straylight outside the field of view that will be further scattered by the objective housing.

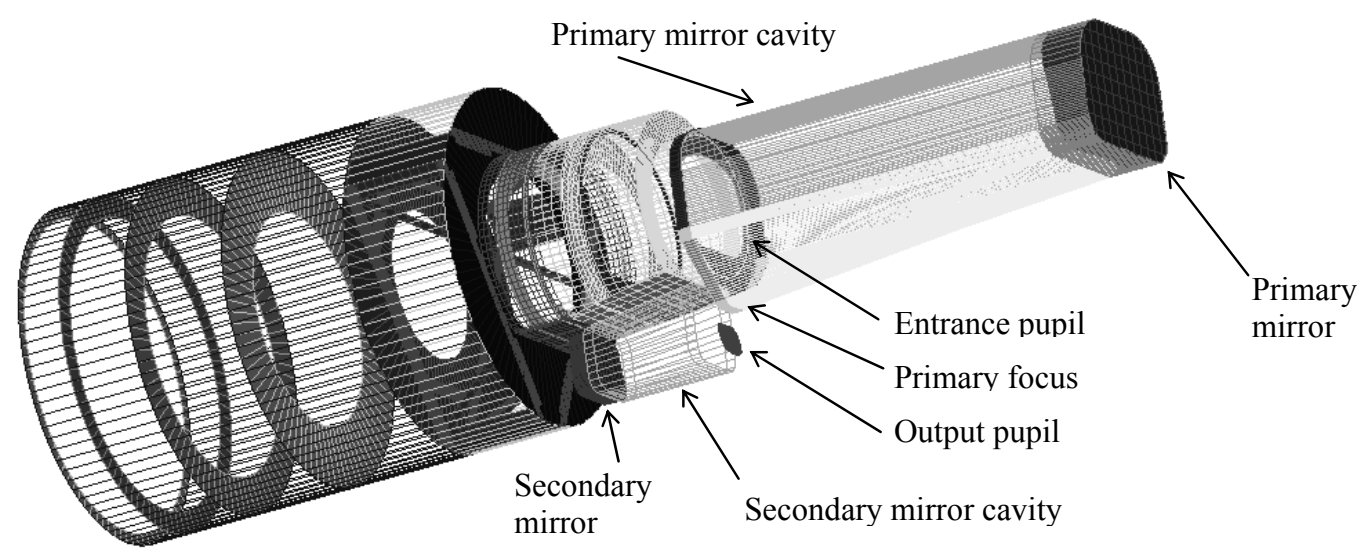

Figure 7: ASAP model for the analysis of the straylight analysis.

Different coatings have been modeled by their correspondent BRDF i.e. Aeroglaze Z306 for the baffle and black anodized aluminum for the mirror cavities. The definition of the curves used is in a first approximation using data found in literature. The mirror roughness and contamination (baseline $2000 \mathrm{ppm}$ ) has also been modeled by their respective BRDF. Roughness modeling is based on previous measurement on representative samples and the BRDF caused by contamination has been theoretically computed using Mie scattering theory.

\subsection{ASAP analysis}

The main drawback of ASAP is the very high number of rays that have to be created to reach a good accuracy on the result after several scattering. Hopefully, the number of rays has been reduced using new possibilities in ASAP and the sequential aspect of the system. The baffle has been divided in 8 sections. At the end of a section, a virtual detector has been placed to catch the rays going to the next section. Once the computation is complete in a section, the next one started with a source being the rays caught at previous detector. This allows a high number of scatterings for the useful rays while limiting to a maximum the useless rays.

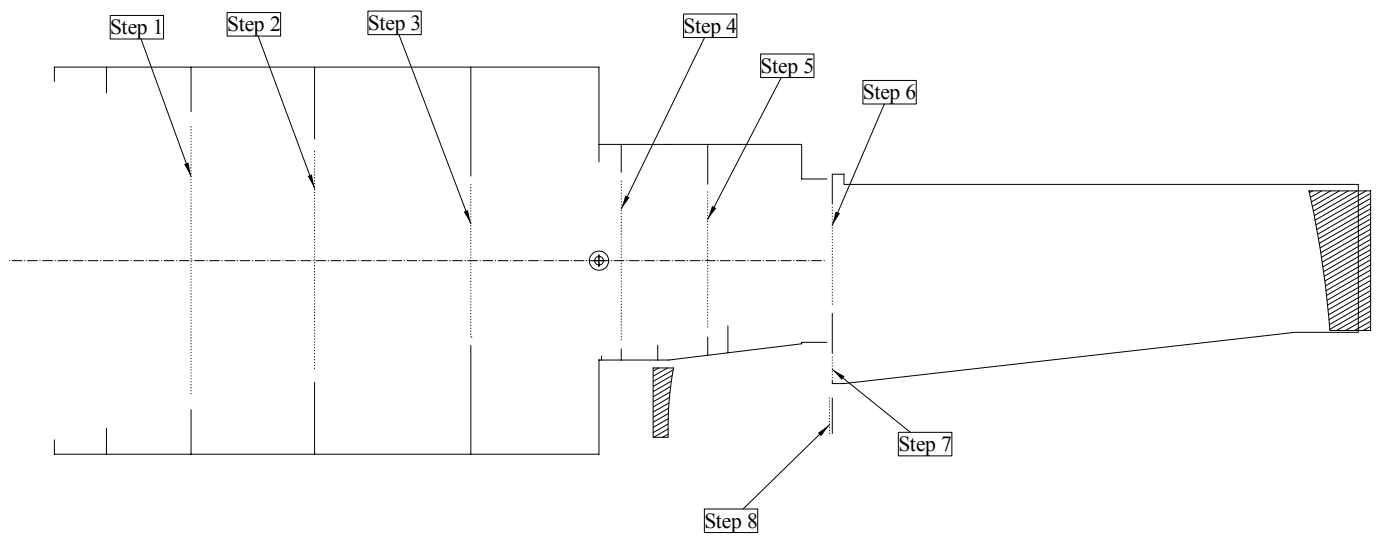

Figure 8: Model profile and separation in sections 


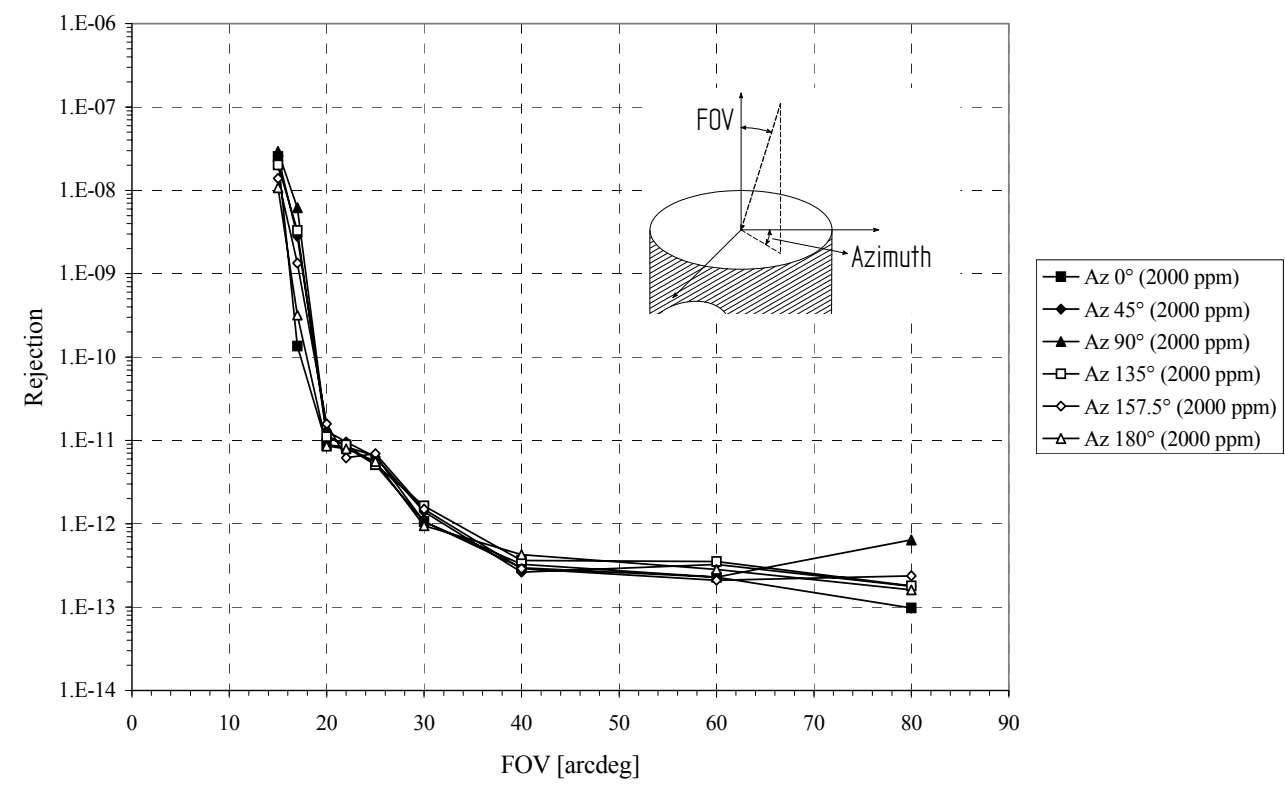

Figure 9: Results of the straylight analysis

Figure 9 presents the level of straylight in the field of view of the dioptric objective. The included drawing representing the aperture of the baffle defines the FOV and the azimuth.

These curves indicate a good symmetry of the baffle above 20 degrees. The curves also reflect the geometry of the baffle: below $20^{\circ}$, external light directly enters into the mirror tube (FOV of the transition depends on the azimuth due to asymmetry of the pupil). Up to $27^{\circ}$, the light enters the smaller baffle tube, up to $32^{\circ}$, the light goes through the vane 1 (first stage). Above this value, the rejection is higher than $10^{12}$.

These results have been used by the scientific community to determine the final level of straylight reaching the detector. According to these results, the selection of targets has been initiated.

\subsection{Edges diffraction}

Some computations have been performed to determine the level of straylight caused by the diffraction on the critical edges (mainly vane 1). Theoretical analysis has determined that the energy diffracted by edge of vane 1 reaching primary mirror was negligible with respect to other sources. The diffraction computation has then been removed from the final results.

\section{6. $\quad$ Edges scattering}

As mentioned earlier, the edges can be considered has critical surfaces as it will never be possible to avoid that some part of it will see primary mirror and be lightened by external light.

Early studies have defined the edges as flat surfaces (parallel to optical axis) with a thickness of $40 \mu \mathrm{m}$. These surfaces are too small to be hit by rays of the global ray-tracing analysis. It was then necessary to perform a dedicated study with ASAP.

A source has been created specifically to lighten one of the edges with a sufficient number of rays and the scattered rays have been oriented preferably towards the primary mirror. Figure 10 represents resulting straylight at the level of the output pupil (FOV of dioptric objective) for all edges except vane 3 and 4. It confirms the first order analysis (Table 1), vanes $1, \mathrm{~s} 2$, s2bis and s3bis are the most critical ones (highest level). Nevertheless, most of that straylight will be negligible compared with the higher levels in the global scattering analysis. Vane 1 is the only one generating a level of straylight comparable to the global one. Figure 11 shows a comparison of both effect (edges straylight has been summed). 
Baffle edge diffusion at output pupil into FOV $\left(\mathrm{Az} 180^{\circ}\right)$

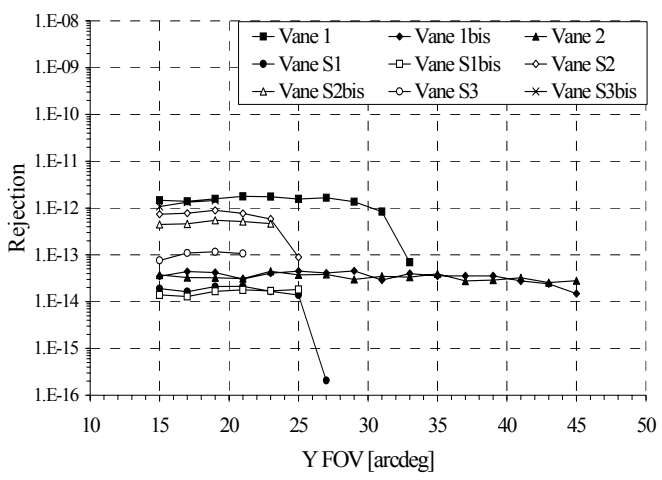

Figure 10: Level of straylight in the FOV of the dioptric objective due to edges scattering
Baffle edge diffusion at output pupil into FOV $\left(\mathrm{Az} 180^{\circ}\right)$

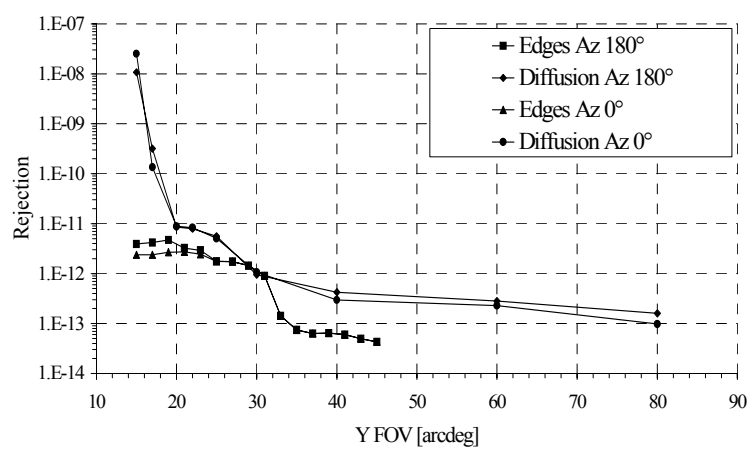

Figure 11: Comparison of levels due to global scattering and due to edges

\subsection{Sensitivity to hypotheses}

Before concluding, some analyses have been performed to evaluate the sensitivity to the hypotheses and the statistical accuracy.

The first point was to determine the number of rays in the first source grid located at the entrance of the baffle. The number of rays will directly determine the duration of calculation but also the pitch of the grid and thus the size of the smallest seen object. The baseline selected size was 171 x 171 (one ray per $20 \mathrm{~mm}^{2}$ ). Several runs were performed for one azimuth and one field of view with different grid size and the result is shown on Figure 12. From $101 \mathrm{x} 101 \mathrm{to} 311 \mathrm{x}$ 311 no important variation is observed. The thickness of the edges is another important point of the model, moreover when we will have to go for the manufacturing. Computations have been performed with $100 \mu \mathrm{m}, 40 \mu \mathrm{m}$ and $10 \mu \mathrm{m}$ edges. Results clearly indicate that the straylight due to the edges is directly proportional to the thickness.

Sensitivity to contamination of the mirrors will also define the margins we have on the contamination budget for the telescope and the baffle. The 2000-ppm is the value after launch as estimated by a contamination budget. Calculation with other contamination on the mirrors has been performed. Once again, a proportional behavior is observed (see Figure 13).

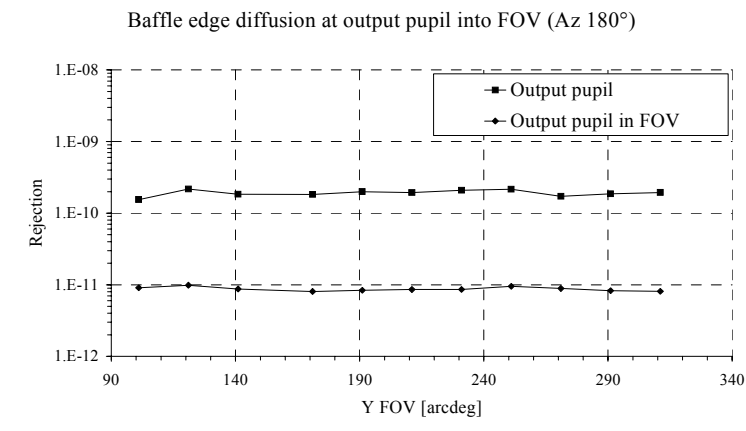

Figure 12: Sensitivity to grid size

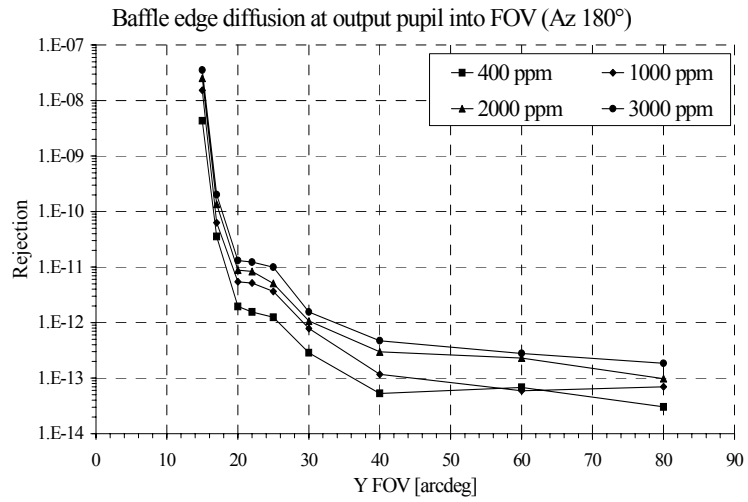

Figure 13: Sensitivity to mirrors contamination

Finally, several computations were performed at the same azimuth and field of view but changing each times the statistical parameters in ASAP. The variation of the final result stays within $15 \%$ of the average result. This variation can be considered small with respect to the factor 2 generally considered for the accuracy of the result of such an analysis. 


\subsection{Conclusions}

We can conclude that the proposed baffle has a global rejection close to the one required. The edges (mainly vane 1 edge) are considered critical and will be designed carefully. Below $20^{\circ}$, the rejection is driven by the primary mirror cavity and its coating. Between $20^{\circ}$ and $27^{\circ}$ the rejection is driven by the small tube, between $27^{\circ}$ and $32.5^{\circ}$, the rejection is driven by the cavity between vanes $\mathrm{s} 1$ and 1 and above $32.5^{\circ}$, rejection is driven by the baffle up to vane 1 .

The sensitivity analysis shows that the results of the computations can be considered accurate (with the classical factor 2). Major unknowns in the model are the coatings and the edges.

Further analysis at this point by the scientific community shows that the resulting attenuation is acceptable but it is also emphasized that, as far as possible, the real design should not be worst than the computation so that the off pointing possibilities are kept large enough. The major risk is identified below $20^{\circ}$.

\section{MECHANICAL DESIGN}

\subsection{Structural design}

The proposed solution is an aluminum baffle. The initial major constraints are the first eigenfrequency (above $150 \mathrm{~Hz}$ ), the mass (under $17 \mathrm{~kg}$ ) and the transmitted forces to the telescope due to differential thermal expansion.

To limit conduction and transmitted forces, the baffle is maintained on the telescope by three flexible legs (blades) made of titanium (see Figure 16). These legs have been also optimized to keep the eigenfrequency as high as possible.

In order to improve also the stiffness of the complete structure, Tulip shape structure has been optimized for the main tube. The bottom of the tube is $0.8 \mathrm{~mm}$ thick while the upper part is $0.4 \mathrm{~mm}$. The separation between both areas is a wave so that in three position (above the legs), the 0.8 wall goes nearly to the top. This improves the stiffness while reducing the mass (see Figure 14).

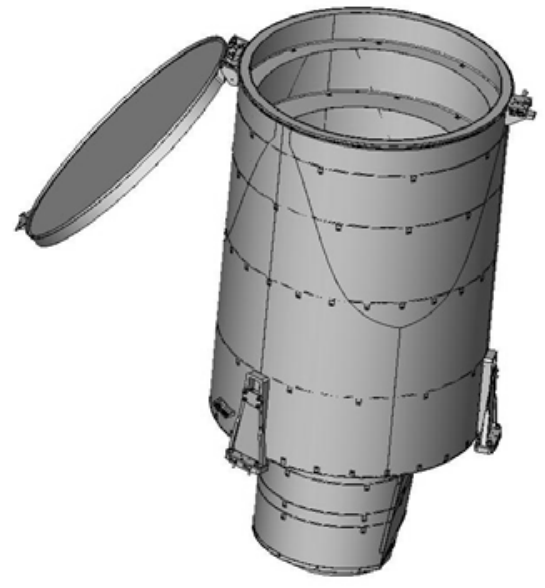

Figure 14: View of the COROT baffle with cover. Tulip shaped structure on the tube is visible

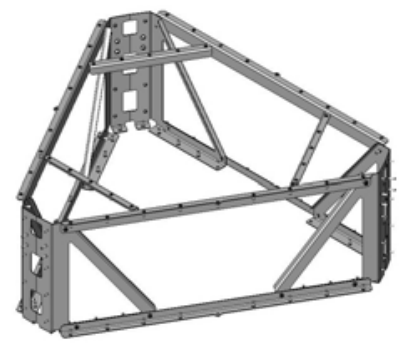

Figure 15: Internal structure located between vanes $\mathrm{s} 1$ and 1 to stiffen the baffle

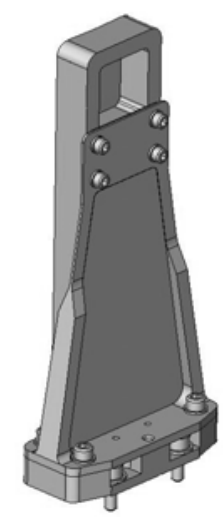

Figure 16: Close view of one of the mounting feet

Strong effort has been put to further improve the structure since the resulting design leads to a too low eigenfrequency. An internal structure (Figure 15) has been added between vane s1 and vane 1 to suppress a low eigenfrequency mode that can perturb the telescope. At the moment, the structural design is still subject to modification depending on coupled analysis with telescope.

\subsection{Painting}

The internal geometry of the baffle after mechanical design respects in most areas the optical design. Some screws and brackets are added for fixation of the vanes. The most important change is the internal stiffening structure. This structure 
has been correctly hidden in the cavity and is not a critical element but it will be included in an updated straylight analysis.

The important factor for the straylight analysis is also the choice of coating. The use of aluminum suggests the use of black anodization. Some measurements indicate that the absorption of that coating could not reach the modeled one. Improvement could have been reached by sandblasting the aluminum sheet. This solution has been considered too risky due to the very low thickness of the sheet.

The choice has thus been made to use Aeroglaze Z306 which is a well known paint for space application. Measurement made on samples indicates that BRDF was close to the modeled one.

\subsection{Edges design}

Second critical point of the mechanical design was the manufacturing of the edges. Specification required a flat edge of $40 \mu \mathrm{m}$. Different solutions have been analyzed: stainless steel foil cut by laser or by waterjet, milled aluminum with or without paint.

The stainless steel sheet was one of the solutions, the problem was to cut the circular aperture. Both laser and waterjet techniques were tested. Laser cutting was rapidly rejected because the process creates at the edge a large local increase of thickness. The waterjet cutting resulted in a very rough edge, inducing no uniformity of the edge behavior depending on the measured location (see Figure 17). The main drawback of the stainless steel foil was also the impossibility to blacken the foil because of its very low thickness. The second solution envisaged is a milled aluminum sheet: starting from a 0.4$\mathrm{mm}$ sheet, the aperture was milled using a cutting angle of $15^{\circ}$. The resulting edge was of very good quality. Starting from a painted edge, this process results in a large shinny surface ( $2 \mathrm{~mm}$ in the case of 0.4 -sheet), moreover, the cutting of the paint could have caused adhesion problems (see Figure 19).

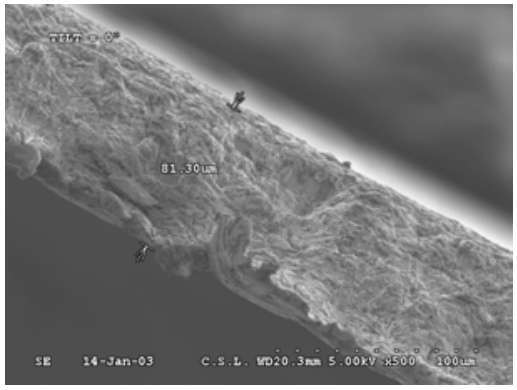

Figure 17: Waterjet cut stainless steel foil

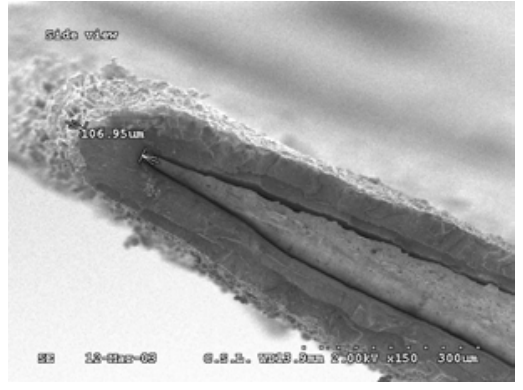

Figure 18: Milled then painted aluminum sheet

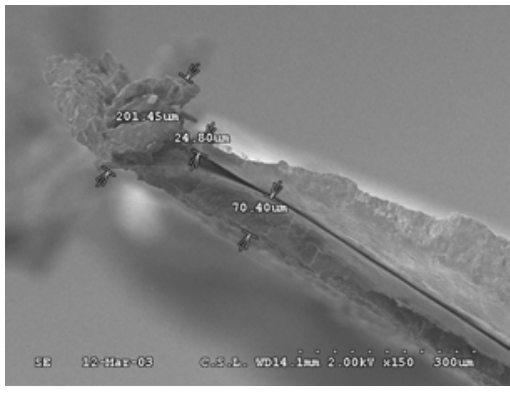

Figure 19: Painted then milled aluminum sheet

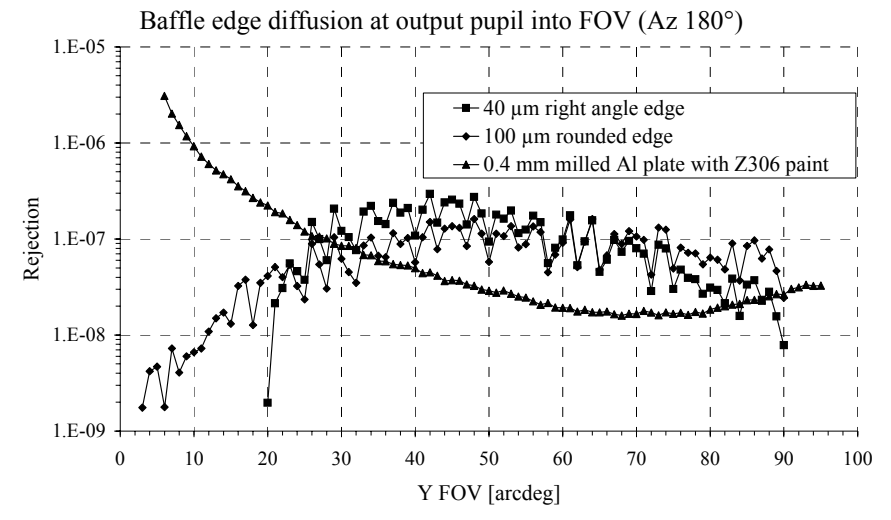

Figure 20: Comparison of measurement with computation
Another test has been performed with a sample first milled then painted (see Figure 18). The resulting thickness was higher (radius of $70 \mu \mathrm{m}$ ) and it was necessary to compare the obtained results with the original modeling of the edges. A small ASAP model has been built representing the test set-up. The $40 \mu \mathrm{m}$ flat edge and a $100 \mu \mathrm{m}$ rounded edges were modeled. Figure 20 shows the comparison between the different results. $40 \mu \mathrm{m}$ flat and $100 \mu \mathrm{m}$ rounded give similar results and the measured edge is of the same order of magnitude. This last solution has been chosen as baseline. 


\section{UPDATE OF STRAYLIGHT ANALYSIS}

After the detailed design review of all the elements included in the optical model, an update of the straylight analysis model will be performed. It will include all the elements whose mechanical design could not fully respect the optical design. It includes coatings, edges thickness, internal stiffening structure of the baffle and some dimensions.

All elements have up to now been analysed one by one without important impact on the straylight. The global analysis will confirm this.

\section{CONCLUSIONS}

This paper describes the straylight analysis performed on the baffle. This baffle goal is to attenuate straylight for fields higher than $20^{\circ}$ in order to eliminate the periodic perturbation on measurements. This straylight is mainly coming from Earth albedo.

A first order verification has been done in order to detect critical surfaces that could not be detected by the global model. It allowed also performing a selection of the points that required special attention (e.g. edges)

The analysis has been performed according to a technique that allows high accuracy computation on a large structure to very high rejection factors. The results of the analysis show a good correlation with the geometry of the baffle.

Then the mechanical design is shortly discussed with some attention on the points considered critical with respect to straylight. Finally, the updated model is introduced.

\section{AKNOWLEDGMENT}

The Belgian contribution to the COROT program is funded by the Federal Office for Scientific, Technical and Cultural Affairs (OSTC), in the frame of the ESA/Prodex program.

\section{REFERENCES}

List of papers about COROT can be found on http://www.astrsp-mrs.fr/projets/corot/arti corot.html.

1. F. Paoli, F. Douillet, M. Jouret-Perl, J.B. Dubois, "COROT: a small satellite in low earth orbit for asteroseismology and the search of exoplanets", 50 ${ }^{\text {th }}$ International Astronautical Congress, 4-8 Oct 1999, Amsterdam, The Netherlands, IAF-99-Q.1.02.

2. A. Baglin, M. Auvergne, C. Catala, E.Michel and the Corot Team, "Asteroseimology with the Space mission COROT: photometric performances, targets and mission profile", SOHO/GONG workshop, Tenerife, October 2000 